\title{
AMBIENTE DE TRABALHO SAUDÁVEL NA ATENÇÃO PRIMÁRIA À SAÚDE: REVISÃO INTEGRATIVA DA LITERATURA
}

\author{
HEALTHY WORK ENVIRONMENT IN \\ PRIMARY HEALTH CARE: INTEGRATIVE \\ LITERATURE REVIEW
}

\section{AMBIENTE DE TRABAJO SALUDABLE EN ATENCIÓN PRIMARIA: REVISIÓN BIBLIOGRÁFICA INTEGRADORA}

\author{
Lenna Eloisa Madureira Pereira ${ }^{1}$ \\ Flavia Regina Souza Ramos ${ }^{2}$ \\ Laura Cavalcanti de Farias Brehmer ${ }^{3}$ \\ Paola da Silva Diaz ${ }^{4}$
}

\begin{abstract}
Como citar este artigo: Pereira LEM, Ramos FRS, Brehmer LCF, Diaz PS. Ambiente de trabalho saudável na Atenção Primária à Saúde: revisão integrativa da literatura. Rev baiana enferm. 2022:36:e38084.

Objetivo: identificar, na produção científica, elementos constituintes do ambiente de trabalho saudável na Atenção Primária à Saúde para a saúde dos trabalhadores. Método: revisão integrativa da literatura, realizada no período de junho a agosto de 2019, conforme protocolo de busca construído com parâmetros de elegibilidade para a definição de uma amostra de 35 estudos, para responder à questão norteadora: Quais elementos constituintes do ambiente de trabalho na atenção primária referem-se à saúde dos trabalhadores? Resultados: a análise resultou na elaboração das categorias acerca do adoecimento e do estresse como repercussões da vivência subjetiva do trabalho e das alternativas protetivas, de educação e de transformação dos ambientes de trabalho. Conclusão: na produção científica estudada, os elementos constituintes do ambiente de trabalho saudável na Atenção Primária à Saúde, relacionados à saúde dos trabalhadores, são geradores de adoecimento e estresse e estão em constante associação ao contexto desse nível de atenção, à gestão política e do processo de trabalho na saúde e às vivências subjetivas no trabalho.
\end{abstract}

Descritores: Ambiente de trabalho. Saúde do trabalhador. Atenção Primária à Saúde. Saúde pública. Enfermagem do Trabalho.

Objective: to identify, in the scientific production, constituent elements of a bealthy work environment in Primary Health Care for workers' bealth. Method: integrative literature review, conducted from June to August 2019, according to a search protocol built with eligibility parameters for the definition of a sample of 35 studies, to answer the guiding question: Which elements of the work environment in primary care refer to workers' bealth? Results: the analysis resulted in the elaboration of categories about illness and stress as repercussions of the subjective experience of work

\footnotetext{
Enfermeira. Mestre em Enfermagem. Universidade Federal de Santa Catarina. Florianópolis, Santa Catarina, Brasil. lenna.madureira@gmail.com. http://orcid. org/0000-000 I-9326-0538.

2 Enfermeira. Doutora em Enfermagem. Professora Titular da Universidade Federal de Santa Catarina. Florianópolis, Santa Catarina, Brasil. http://orcid.org/0000-00020077-2292.

3 Enfermeira. Doutora em Enfermagem. Professora da Universidade Federal de Santa Catarina. Florianópolis, Santa Catarina, Brasil. http://orcid.org/0000-000 I-99658811 .

${ }^{4}$ Enfermeira. Doutora em Enfermagem. Universidade Federal de Santa Catarina. Florianópolis, Santa Catarina, Brasil. http://orcid.org/0000-0003-2350-2655.
} 
and the protective alternatives, education and transformation of work environments. Conclusion: in the scientific production studied, the constituent elements of a healthy work environment in Primary Health Care, related to workers' health, are generators of illness and stress and are in constant association with the context of this level of care, with the political management and the health work process, and with subjective experiences at work.

Descriptors: Working Environment. Occupational Health. Primary Health Care. Public Health. Occupational Health Nursing.

Objetivo: identificar, en la producción científica, los elementos constitutivos de un ambiente de trabajo saludable en Atención Primaria de la Salud para la salud de los trabajadores. Método: revisión bibliográfica integradora, realizada de junio a agosto de 2019, según un protocolo de búsqueda construido con parámetros de elegibilidad para la definición de una muestra de 35 estudios, para responder a la pregunta guía: ¿Qué elementos constitutivos del ambiente de trabajo en atención primaria hacen referencia a la salud de los trabajadores? Resultados: el análisis dio lugar a la elaboración de categorias sobre la enfermedad y el estrés como repercusiones de la experiencia subjetiva del trabajo y las alternativas de protección, educación y transformación de los ambientes de trabajo. Conclusión: en la producción científica estudiada, los elementos constitutivos del ambiente de trabajo saludable en la Atención Primaria de Salud, relacionados con la salud de los trabajadores, son generadores de enfermedad y estrés y están en constante asociación con el contexto de este nivel de atención, la gestión política y el proceso de trabajo en salud y las experiencias subjetivas en el trabajo.

Descriptores: Ambiente de Trabajo. Salud Laboral. Atención Primaria de Salud. Salud Pública. Enfermería del Trabajo.

\section{Introdução}

Compreende-se por ambiente de trabalho saudável aquele no qual todos os trabalhadores colaboram coletivamente, em um processo de melhoria contínua da proteção e promoção da segurança, saúde e bem-estar de todos, com o apoio da gestão. Esta definição, elaborada pela Organização Mundial da Saúde (OMS), mediante consulta a diferentes organizações, ao incluir fatores psicossociais e práticas de saúde, revela uma crescente preocupação com os ambientes de trabalho, por sua amplitude e relação com a promoção da saúde ${ }^{(1)}$.

A própria OMS enfatiza a necessidade de pesquisas e estudos dentro do ambiente da prática profissional em instituições de saúde, tendo em vista a percepção da lacuna existente ${ }^{(2)}$. Na Atenção Primária à Saúde (APS), assim como nos diferentes níveis de atenção, considera-se o fato de os elementos integrantes do ambiente de trabalho oferecerem riscos tanto para a segurança do público como de seus trabalhadores. O conjunto desses elementos, assim como as relações que estabelecem entre si, pode constituir instrumento de análise e interpretação das condições e qualidades desses ambientes.
Seja por meio de instrumentos de avaliação do ambiente de trabalho, seja por meio de estudos com distintos enfoques metodológicos sobre a temática, as contribuições podem implicar em ações voltadas ao trabalhador. As produções científicas conferem visibilidade às condições de trabalho que repercutem sobre a saúde do trabalhador, e as análises, potencialmente, descortinam macro e microcenários laborais ${ }^{(3)}$.

$\mathrm{Na}$ Atenção Primária, pode-se elencar alguns fatores que interferem sobre a saúde do trabalhador, relacionados às excessivas e complexas cargas de trabalho e às condições de trabalho causadoras de adoecimento físico e emocional $^{(4-5)}$. A APS, primeiro nível de contato das pessoas com os Sistemas de Saúde, na maioria dos casos de demandas nessa área, é a porta de entrada e ordenadora dos fluxos e contrafluxos entre todos os pontos da rede de atenção à saúde ${ }^{(6)}$. Passados 30 anos da implantação do Sistema Único de Saúde (SUS), discutir o ambiente de trabalho saudável na Atenção Primária brasileira insere esse contexto nas pautas globais sobre a saúde do trabalhador, situando limites históricos e culturais próprios. 
O conceito de ambiente de trabalho saudável implicado no contexto da APS possibilitou o desenho da questão norteadora desta pesquisa: "Quais elementos constituintes do ambiente de trabalho na Atenção Primária referem-se à saúde dos trabalhadores?" O objetivo foi identificar, na produção científica, elementos constituintes do ambiente de trabalho na Atenção Primária para a saúde dos trabalhadores.

\section{Método}

Trata-se de um estudo bibliográfico do tipo Revisão Integrativa da Literatura cujos processos de análise e categorização foram organizados criteriosamente em seis fases ${ }^{(7)}$ : fase 1 - estabelecimento da questão de pesquisa; fase 2 - busca da literatura nas bases de dados; fase 3 - categorização dos estudos; fase 4 - avaliação dos estudos incluídos na revisão; fase 5 - interpretação dos resultados; e fase 6 - síntese do conhecimento.

O protocolo de revisão de literatura foi elaborado no período de junho a agosto de 2019 com a questão norteadora: Quais elementos constituintes do ambiente de trabalho na Atenção Primária referem-se à saúde dos trabalhadores? A delimitação temporal foi fixada entre 2010 e abril de 2019. O tipo de publicação foi artigo. As estratégias de combinações entre 109 termos de busca alternativos e sinônimos relacionados aos Descritores em Ciências da Saúde (DeCS) referiram-se a quatro temas: Ambientes de trabalho, Atenção Primária à Saúde, Fator associado ao ambiente e à saúde do trabalhador. Foram estabelecidos os idiomas inglês, espanhol e português. As bases de dados consultadas foram: Publisher Medline (PubMed), Cumulative Index to Nursing \& Allied Health Literature (CINAHL), Scientific Electronic Library Online (SciELO), Sci Verse Scopus (Elsevier), Web of Science (WoS),
Literatura Latino-Americana e do Caribe em Ciências da Saúde (LILACS) e Base de Dados de Enfermagem (BDENF).

A opção pelo ano de 2010, como data inicial da pesquisa, deve-se à divulgação do marco conceitual referente à saúde dos trabalhadores, publicada pela OMS, que considera um plano de ação global com objetivos de promoção e proteção da saúde do trabalhador no ambiente de trabalho ${ }^{(1)}$.

Para o gerenciamento das fontes, foi usado o software Mendeley. Para evitar viés de interpretação, dois revisores realizaram a seleção e categorização dos resultados simultânea e independentemente. Ambos seguiram o protocolo de revisão, com leitura minuciosa e exaustiva de títulos, resumos e textos na íntegra. Um terceiro revisor participou da discussão relativa à resolução de dúvidas e/ou discordâncias. A amostra inicial possibilitou a retirada dos estudos repetidos. Após a leitura de títulos e resumos, ocorreram exclusões, por incompatibilidade de conteúdo com os objetivos desta revisão. Na etapa de leitura na íntegra dos estudos, novas exclusões foram feitas, pois se constatou que alguns não respondiam à questão norteadora.

Elaborou-se uma planilha de dados no software Microsoft Excel ${ }^{\circledR}$, contendo as seguintes informações: autor(es), ano de publicação, idioma, país de origem, periódico de publicação, delineamento do estudo, tema principal do artigo. O conteúdo dos artigos foi inserido no software Atlas.ti 7.5.18, para a organização de informações, com identificação dos códigos correspondentes aos elementos constituintes do ambiente de trabalho saudável na atenção primária à saúde e agrupados posteriormente, por similaridade temática, para o delineamento das categorias. A Figura 1 ilustra o processo de seleção e definição das amostras. 
Figura 1 - Processo de busca e definição das amostras da Revisão Integrativa da Literatura

\section{Quais elementos constituintes do ambiente de trabalho na atenção primária se referem à saúde dos trabalhadores?}
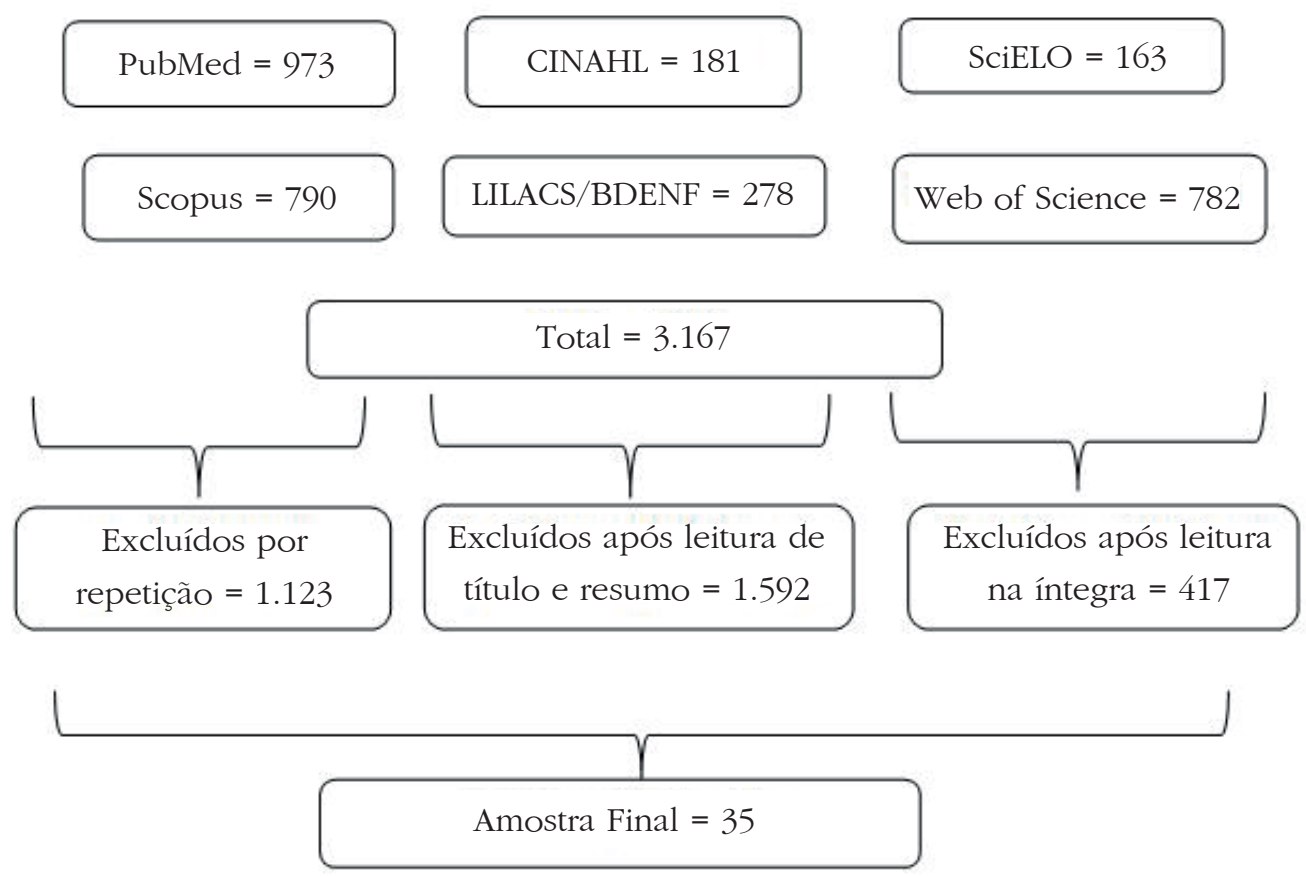

Fonte: Elaboração própria.

\section{Resultados}

Dos 35 artigos que compuseram a amostra deste estudo, o maior número de publicações ocorreu no período de 2013 a 2015 (51,4\%), seguidos de 2016 a 2018 (31,4\%). Os artigos foram publicados nos idiomas inglês $(51,4 \%)$, português brasileiro (45,7\%) e espanhol (2,9\%). Trabalhos com métodos quantitativos (63\%) configuraram-se como líderes, seguidos dos qualitativos (34,2\%); com parcela mínima veem-se trabalhos de métodos mistos (2,8\%). Os estudos incluídos na presente revisão são apresentados de acordo com: autor(es), idioma de publicação e país de origem; periódico e ano de publicação; e recorte do tema de interesse para a revisão (Tabela 1).

Tabela 1 - Artigos da amostra segundo idioma de publicação, método e ano de publicação. Florianópolis, Santa Catarina, Brasil - 2010-2019 (N=35)

\begin{tabular}{l|c|c}
\hline Variáveis & $\mathbf{n}$ & $\mathbf{\%}$ \\
\hline Idioma de publicação & & 51,4 \\
$\quad$ Inglês & 18 & 2,9 \\
Espanhol & 1 & 45,7 \\
$\quad$ Português & 16 & 63 \\
Método & & 34,2 \\
$\quad$ Quantitativo & 22 & 2,8 \\
Qualitativo & 12 & \\
Misto & 1 & 14,3 \\
Ano de publicação & & 51,4 \\
2010-2012 & 5 & 31,4 \\
$2013-2015$ & 18 & 2,9 \\
$2016-2018$ & 11 & \\
2019 & 1 & \\
\hline
\end{tabular}

Fonte: Elaboração própria. 
Os resultados foram agrupados e discutidos em duas categorias: 1 - $\mathrm{O}$ adoecimento e o estresse como repercussões da vivência subjetiva do trabalho; e 2 - Alternativas protetivas, educação e transformação dos ambientes de trabalho.
O Quadro 1 apresenta os artigos da amostra, separados por autor e ano de publicação, foco de interesse e categorias de análise.

Quadro 1 - Artigos da amostra segundo autor(es) e ano de publicação, foco de interesse e enquadramento por categoria(s) de análise

(continua)

\begin{tabular}{|c|c|c|}
\hline Autoria e ano de publicação & Foco de interesse & $\begin{array}{l}\text { Categorias } \\
\text { de análise }\end{array}$ \\
\hline $\begin{array}{l}\text { Peralta N, Godoi Vasconcelos AG, } \\
\text { Griep RH, Miller L. } 2012^{(8)}\end{array}$ & $\begin{array}{l}\text { Índice de Capacidade para o Trabalho (TIC) } \\
\text { na Atenção Primária à Saúde. }\end{array}$ & 1,2 \\
\hline Halcomb E, Ashley C. $2016^{(9)}$ & $\begin{array}{l}\text { Força de trabalho de enfermagem, } \\
\text { satisfação e estratégias para a sua retenção } \\
\text { no ambiente. }\end{array}$ & 1 \\
\hline $\begin{array}{l}\text { Abbas MAF, Fiala LA, Rahman AGA, } \\
\text { Fahim ARE. } 2010^{(10)}\end{array}$ & $\begin{array}{l}\text { Violência contra profissionais de saúde no } \\
\text { local de trabalho. }\end{array}$ & 1 \\
\hline $\begin{array}{l}\text { Pinto ACS, Almeida MI, Pinheiro PNC. } \\
2011^{(11)}\end{array}$ & $\begin{array}{l}\text { Status vacinal de profissionais na Atenção } \\
\text { Primária à Saúde e vulnerabilidade a } \\
\text { riscos biológicos. }\end{array}$ & 1 \\
\hline Dias MDA. $2013^{(12)}$ & $\begin{array}{l}\text { A integralidade da atenção em Saúde do } \\
\text { Trabalhador e expansão de ações para a } \\
\text { Atenção Primária à Saúde. }\end{array}$ & 1 \\
\hline $\begin{array}{l}\text { Bertussi VC, Junqueira MABB, } \\
\text { Giuliani CD, Calçado RM, Miranda FJS, } \\
\text { Santos MA, et al. } 2018^{(13)}\end{array}$ & $\begin{array}{l}\text { Associação entre uso de substâncias } \\
\text { psicoativas e depressão, estresse } \\
\text { e ansiedade entre profissionais de } \\
\text { enfermagem na Atenção Primária à Saúde. }\end{array}$ & 1 \\
\hline $\begin{array}{l}\text { González Jara MA, Mora Hidalgo A, } \\
\text { Avalos Gulin JC, Albiach ML, Ortiz LM, } \\
\text { Monserrat PT, et al. } 2013^{(14)}\end{array}$ & $\begin{array}{l}\text { Exposição de compostos químicos } \\
\text { glutaraldeído na Atenção Primária à Saúde. }\end{array}$ & 1 \\
\hline $\begin{array}{l}\text { Siegrist J, Shackelton R, Link C, } \\
\text { Marceau L, Knesebeck O, Mckinlay J. } \\
2010^{(15)}\end{array}$ & $\begin{array}{l}\text { Níveis de estresse relacionado ao trabalho } \\
\text { entre profissionais da Atenção Primária à } \\
\text { Saúde em diferentes sistemas de saúde. }\end{array}$ & 1 \\
\hline $\begin{array}{l}\text { Oliveira LP, Camargo FC, Iwamoto HH. } \\
2013^{(16)}\end{array}$ & $\begin{array}{l}\text { Violência no cotidiano de trabalho das } \\
\text { equipes de saúde da família. }\end{array}$ & 1,2 \\
\hline $\begin{array}{l}\text { Gómez-Gascón T, Martín-Fernández } \\
\text { J, Gálvez-Herrer M, Tapias-Merino E, } \\
\text { Beamud-Lagos M, Mingote-Adán JC, } \\
\text { et al. } 2013^{(17)}\end{array}$ & $\begin{array}{l}\text { Síndrome de Burnout e alternativas } \\
\text { organizacionais para a prevenção. }\end{array}$ & 2 \\
\hline $\begin{array}{l}\text { Costa PCC, Francischetti-Garcia APRF, } \\
\text { Pellegrino-Toledo VP. } 2016^{(18)}\end{array}$ & $\begin{array}{l}\text { Expectativas de enfermeiros brasileiros em } \\
\text { relação à forma de acolhimento realizado } \\
\text { por eles na Atenção Primária em Saúde. }\end{array}$ & 1 \\
\hline $\begin{array}{l}\text { Hongxia Guo RN, Chunping Ni RN, } \\
\text { Changqing Liu RN, Jiping Li BN, } \\
\text { Suzhen Liu MSN. 2019 }\end{array}$ & $\begin{array}{l}\text { Estresse e influências percebidas entre } \\
\text { enfermeiros na atenção comunitária. }\end{array}$ & 1 \\
\hline $\begin{array}{l}\text { Silva MO, Peixoto DA, Nóbrega TBT. } \\
2013^{(20)}\end{array}$ & $\begin{array}{l}\text { Percepção da enfermagem sobre acidente e } \\
\text { condições de saúde na atenção primária. }\end{array}$ & 1 \\
\hline $\begin{array}{l}\text { Cipriano FG, Ferreira LP, Servilha EAM, } \\
\text { Marsiglia RMG. } 2013^{(21)}\end{array}$ & $\begin{array}{l}\text { Distúrbio de voz e rotina de trabalho } \\
\text { de Agentes Comunitários de Saúde no } \\
\text { ambiente e na organização do trabalho. }\end{array}$ & 1 \\
\hline $\begin{array}{l}\text { Silveira SLM, Câmara SG, Amazarray MR. } \\
2014^{(22)}\end{array}$ & $\begin{array}{l}\text { Prevalência e preditores da Síndrome de } \\
\text { Burnout entre trabalhadores. }\end{array}$ & 1 \\
\hline
\end{tabular}


Quadro 1 - Artigos da amostra segundo autor(es) e ano de publicação, foco de interesse e enquadramento por categoria(s) de análise

(continuação)

\begin{tabular}{|c|c|c|}
\hline Autoria e ano de publicação & Foco de interesse & $\begin{array}{l}\text { Categorias } \\
\text { de análise }\end{array}$ \\
\hline $\begin{array}{l}\text { Scherer MDA, Oliveira CI, } \\
\text { Carvalho WMES, Costa MP. } 2016^{(23)}\end{array}$ & $\begin{array}{l}\text { Desconexão entre trabalho e formação e } \\
\text { a ferramenta da educação via especialização. }\end{array}$ & 1 \\
\hline $\begin{array}{l}\text { Silva SCPS, Nunes MAP, Santana VR, } \\
\text { Reis FP, Machado Neto J, Lima SO. } \\
2015^{(24)}\end{array}$ & $\begin{array}{l}\text { Prevalência e fatores associados à Síndrome } \\
\text { de Burnout em profissionais da Atenção } \\
\text { Primária à Saúde. }\end{array}$ & 1,2 \\
\hline $\begin{array}{l}\text { Speroni KS, Fruet IMA, Dalmolin GL, } \\
\text { Lima SBS. } 2016^{(25)}\end{array}$ & $\begin{array}{l}\text { Percepções e motivações de Agentes } \\
\text { Comunitários de Saúde sobre suas ações no } \\
\text { processo de trabalho. }\end{array}$ & 1 \\
\hline $\begin{array}{l}\text { Pegoraro PBB, Schaefer R, Zoboli } \\
\text { ELCP. } 2017^{(26)}\end{array}$ & $\begin{array}{l}\text { Identificação, gerenciamento e prevenção } \\
\text { do esgotamento psíquico e moral de } \\
\text { trabalhadores da atenção primária. }\end{array}$ & 1,2 \\
\hline $\begin{array}{l}\text { Costa FM, Martins AMEBL, Lima CA, } \\
\text { Rodrigues QF, Santos KKF, Ferreira RC. } \\
2017^{(27)}\end{array}$ & $\begin{array}{l}\text { Vacinação da Hepatite B entre trabalhadores } \\
\text { da atenção primária. }\end{array}$ & 1 \\
\hline $\begin{array}{l}\text { Zhang M, Yang R, Wang W, Gillespie J, } \\
\text { Clarke S, Yan F. } 2016^{(28)}\end{array}$ & $\begin{array}{l}\text { Satisfação no trabalho dos agentes } \\
\text { comunitários e reformas na atenção da } \\
\text { Atenção Primária à Saúde da China. }\end{array}$ & 1 \\
\hline Nehmy RMQ, Dias EC. 2010 & $\begin{array}{l}\text { Reflexão sobre as mudanças no mundo do } \\
\text { trabalho, reorganização do Sistema Único } \\
\text { de Saúde e prática dos profissionais na } \\
\text { atenção básica. }\end{array}$ & 1 \\
\hline $\begin{array}{l}\text { Maun A, Nilsson K, Furaker C, } \\
\text { Thorn J. } 2013^{(30)}\end{array}$ & $\begin{array}{l}\text { Transição do sistema de saúde e o impacto } \\
\text { percebido no trabalho por gerentes de } \\
\text { centros de saúde. }\end{array}$ & 1 \\
\hline $\begin{array}{l}\text { Bhatnagar A, Gupta S, Alonge O, } \\
\text { George AS. } 2017^{(31)}\end{array}$ & $\begin{array}{l}\text { Fatores individuais e organizacionais, } \\
\text { suas interações e efeitos na motivação } \\
\text { na atenção primária, como } \\
\text { incentivos financeiros. }\end{array}$ & 2 \\
\hline $\begin{array}{l}\text { Fisekovic Kremic MB, Terzic-Supic ZJ, } \\
\text { Santric-Milicevic MM, Trajkovic GZ. } \\
2017^{(32)}\end{array}$ & $\begin{array}{l}\text { Violência verbal e psicológica no local de } \\
\text { trabalho na Atenção Primária à Saúde. }\end{array}$ & 2 \\
\hline Rule J, Dunston R, Solomon N. $2016^{(33)}$ & $\begin{array}{l}\text { Metodologia educativa com trabalhadores } \\
\text { e mudanças e ressignificados na Assistência } \\
\text { Primária à Saúde na Austrália. }\end{array}$ & 2 \\
\hline $\begin{array}{l}\text { Silva CCS, Rodrigues LMC, Silva VKBA, } \\
\text { Silva ACO, Silva VLA, Martins MO. } \\
2013^{(34)}\end{array}$ & $\begin{array}{l}\text { Acidentes e condições de trabalho na } \\
\text { Estratégia Saúde da Família na percepção de } \\
\text { profissionais de Enfermagem. }\end{array}$ & 2 \\
\hline $\begin{array}{l}\text { Pessoa VM, Rigotto RM, Arruda CAM, } \\
\text { Machado MFAS, Machado MMT, } \\
\text { Bezerra MGV. } 2013^{(35)}\end{array}$ & $\begin{array}{l}\text { Desenvolvimento econômico e implicações } \\
\text { no trabalho, no ambiente e na saúde } \\
\text { dos trabalhadores. }\end{array}$ & 2 \\
\hline $\begin{array}{l}\text { Costa JO, Sousa MNA, Feitosa ANA, } \\
\text { Feitosa MO, Assis EV, Custódio PP. } \\
2013^{(36)}\end{array}$ & $\begin{array}{l}\text { Estratégias adotadas pela equipe do } \\
\text { departamento de pessoal para minimizar os } \\
\text { conflitos entre trabalhadores nas unidades } \\
\text { básicas de saúde. }\end{array}$ & 2 \\
\hline $\begin{array}{l}\text { Carreiro GSP, Ferreira Filha MO, } \\
\text { Lazarte R, Silva AO, Dias MD. } 2013^{(37)}\end{array}$ & $\begin{array}{l}\text { Desgastes mental e psíquico em } \\
\text { trabalhadores da Estratégia Saúde } \\
\text { da Família. }\end{array}$ & 2 \\
\hline $\begin{array}{l}\text { Duffrin C, Diaz S, Cashion M, } \\
\text { Watson R, Cummings D, Jackson N. } \\
2014^{(38)}\end{array}$ & $\begin{array}{l}\text { Fatores que influenciam o desenvolvimento } \\
\text { da força de trabalho em saúde no } \\
\text { ambiente rural. }\end{array}$ & 2 \\
\hline
\end{tabular}


Quadro 1 - Artigos da amostra segundo autor(es) e ano de publicação, foco de interesse e enquadramento por categoria(s) de análise

(conclusão)

\begin{tabular}{|l|l|c|}
\hline Autoria e ano de publicação & \multicolumn{1}{|c|}{ Foco de interesse } & $\begin{array}{c}\text { Categorias } \\
\text { de análise }\end{array}$ \\
\hline Ojakaa D, Olango S, Jarvis J. 2014 & $\begin{array}{l}\text { (39) } \\
\text { Condições de saúde e desempenho da força } \\
\text { de trabalho em saúde. }\end{array}$ & 2 \\
\hline $\begin{array}{l}\text { Rao KD, Ryan M, Shroff Z, Vujicic M, } \\
\text { Ramani S, Berman P. 2013 }\end{array}$ & $\begin{array}{l}\text { Estratégias de recrutamento rural na } \\
\text { atenção primária. }\end{array}$ & 2 \\
\hline $\begin{array}{l}\text { Cahú GRP, Costa SFG, Costa ICP, } \\
\text { Batista PSS, Batista JBV. 2014 }\end{array}$ & $\begin{array}{l}\text { Assédio moral vivenciado por enfermeiros } \\
\text { no ambiente de trabalho. }\end{array}$ & 2 \\
\hline $\begin{array}{l}\text { Holmes ES, Santos SR, Farias JA, } \\
\text { Costa MBS. 2014 }\end{array}$ & $\begin{array}{l}\text { A Síndrome de Burnout e qualidade de vida de } \\
\text { enfermeiros na Assistência Primária à Saúde. }\end{array}$ & 2 \\
\hline
\end{tabular}

Fonte: Elaboração própria.

As categorias são concorrentes, mas complementares. Inicialmente, isso pode soar como uma contradição, mas os estudos revelaram ambientes de trabalho na Atenção Primária promotores de adoecimento e estresse, contudo, apontaram estratégias de enfrentamento.

\section{1 - O adoecimento e o estresse como repercussões da vivência subjetiva do trabalbo}

Os estudos apresentaram fatores que antecediam, geravam ou mesmo aceleravam o adoecimento físico e psicológico/mental do trabalhador da APS. Os problemas psíquicos e transtornos mentais foram apontados como predominantes entre profissionais de saúde, devido a fatores como elevada exigência da chefia ${ }^{(8-9)}$. Entre esses trabalhadores, depressão e ansiedade figuravam entre os transtornos psiquiátricos relacionados às condições de trabalho caracterizadas pela sobrecarga, rotinas exaustivas e permeadas por relações interpessoais conflituosas e promotoras de adoecimento ${ }^{(10-14)}$.

A proximidade dos trabalhadores da APS com a realidade social dos territórios, aliada à escassez de recursos para prestação de um atendimento efetivo e oferta de atenção integral à saúde, produzia sensação de impotência. Além disso, as interações subjetivas nesses cenários podiam tornar-se fontes de adoecimento psíquico ${ }^{(15)}$. Estudos observaram que os trabalhadores de saúde que atuavam em assistência direta aos usuários estavam mais propensos a demonstrar sintomas psíquicos negativos ${ }^{(14,16)}$. A gestão dos serviços e as políticas de saúde também se constituíam em enfrentamentos causadores de adoecimento e estresse, seja relacionados às situações de falta de ação gerencial, seja por atitudes hierárquicas opressoras $^{(17)}$.

Condições econômicas desfavoráveis e exposição a riscos também se configuraram como fatores limitantes e geradores de adoecimento e estresse do trabalhador da APS ${ }^{(18-19)}$. O desconhecimento de doenças e a falta de atenção às situações de riscos foram igualmente elementos de um ambiente não saudável. Apesar de serem trabalhadores da área da saúde, não estavam imunes a esses aspectos ${ }^{(20-21)}$. Havia obstáculos e lacunas que comprometiam a proteção individual do trabalhador de modo efetivo ${ }^{(22)}$. O dado alarmante ficou por conta do alto grau de desconhecimento dos próprios trabalhadores sobre a notificação de casos de acidentes no ambiente ${ }^{(23)}$, além da precariedade da cobertura de assistência ocupacional para esses trabalhadores ${ }^{(24)}$.

Outro aspecto dos processos de trabalho prejudicados pela gestão referiu-se à alta taxa de rotatividade de profissionais. Estudo identificou a incidência de Burnout entre médicos relacionados a esse contexto ${ }^{(24)}$. O desafio redobra quando tais profissionais são dotados de mecanismos de defesa, que mascaram, sob uma normalidade aparente, um processo latente de sofrimento ou desordem psíquica ${ }^{(25)}$.

Os efeitos negativos dos processos de trabalho em ambientes não saudáveis foram pontos de gatilho para o aparecimento de manifestações 
sintomáticas dos trabalhadores, reveladoras de adoecimento e estresse. Estudos evidenciaram o aumento significativo desses quadros ${ }^{(13-14)}$.

No setor público, a Enfermagem configurou-se como uma profissão estressante ${ }^{(19)}$, com excesso de atividades executadas, número reduzido de profissionais, dificuldades de relacionamento entre a própria equipe, redução salarial, dupla ou tripla jornada, vários vínculos de trabalho, entre outros aspectos ${ }^{(26)}$.

A saúde ocupacional entre trabalhadores da APS foi também identificada como negligente em estudo realizado no Município de Montes Claros (MG), que indicou a necessidades de incorporação de medidas de proteção à saúde do trabalhador nos serviços, além de recomendar que as determinações de protocolos em saúde fossem seguidas ${ }^{(27)}$.

Dentre as dificuldades que permeavam o ambiente profissional desses trabalhadores, estudo ${ }^{(22)}$ destacou: o desgaste emocional diante do convívio com pessoas doentes, conflitos familiares, fragilidades pessoais; situações de risco, incluindo de morte, agressão física e violência; falta de reconhecimento e escassez de recursos humanos. As principais queixas físicas decorrentes do estresse foram: dores de cabeça, hipertensão, ansiedade, problemas de coluna lombar, além do desenvolvimento de outras doenças crônicas ${ }^{(22)}$. O fato é que os profissionais de saúde estavam doentes, em sua maioria incapacitados para a função que exerciam ${ }^{(2)}$. Justificou-se a permanência no trabalho pela necessidade do emprego, além da satisfação pessoal de atuar na profissão escolhida; esta condição, por si, era geradora de adoecimento e estresse ${ }^{(29)}$.

O aumento de horas no trabalho e a diminuição do tempo para a vida pessoal e familiar geravam conflitos de papéis e responsabilidades entre vida pessoal e trabalho. Esse desequilibrio relacionava-se ao aumento de problemas psíquicos nesses trabalhadores ${ }^{(30)}$.

\section{2 - Alternativas protetivas, educação e transformação dos ambientes de trabalho}

Para o enfrentamento de situações geradoras de adoecimento e estresse ou, de um modo geral, para fomentar um ambiente de trabalho saudável na APS, estudos evidenciaram a educação continuada e a educação permanente. Espaços de educação, de escuta e apoio ao trabalhador, voltados especificamente para os aspectos comportamentais e emocionais, tornaram-se indispensáveis no mundo do trabalho ${ }^{(0,31)}$. A Política Nacional de Saúde do Trabalhador e Trabalhadora (PNSTT) configura-se instrumento legal que representa a potencial garantia de ambiente de trabalho saudável, apesar dos limites de concretização na $\operatorname{APS}^{(32)}$.

No âmbito individual, estudos apontaram para as ações do próprio trabalhador sobre o ambiente, para produção de efeitos positivos para sua saúde, proporcionando melhorias para si, com organização interna, planejamento com metas e divisão do tempo, além de flexibilidade na resolução de problemas. Também foram relatadas ações do trabalhador sobre si baseadas nas práticas individuais, tais como exercícios físicos, boa alimentação, lazer, relaxamento e apoio terapêutico ${ }^{(26,33)}$.

O fator conhecimento foi relatado como fundamental para a prevenção de doenças. Profissionais com nível superior apresentavam mais condições de enfrentar os males causados pelos conflitos no ambiente de trabalho ${ }^{(16,34-35)}$. Os riscos físicos e psíquicos no ambiente de trabalho podiam ser devidamente compreendidos e trabalhados como objeto de diálogo e intervenção via ações multiprofissionais, interdisciplinares e intersetoriais na área da saúde, com o objetivo primordial de mudanças nos processos de trabalho ${ }^{(8,17)}$. Alternativas de aprendizagem deviam ser construídas e apoiadas na experiência de grupo, incluindo a abordagem da saúde dos próprios profissionais e o entendimento sobre a saúde do trabalhador ${ }^{(36-37)}$. Assim, ações educativas deviam ser dirigidas para o aprimoramento de conhecimentos e práticas de autocuidado, interferindo na motivação para o processo de trabalho ${ }^{(24)}$.

Em diversos contextos, por mais que fosse conhecida a vulnerabilidade de trabalhadores da APS à violência, pouco se tinha investido para reduzir e enfrentar tal problema; as relações horizontais entre trabalhadores e usuários da 
atenção primária podiam refletir-se na melhor qualidade dos atendimentos ${ }^{(38-39)}$.

As medidas de proteção ao trabalhador em saúde perpassavam por vários fatores, tais como: saúde física, mental e proteção social, atividades de educação permanente e continuada, pausas ao longo da jornada, administração de conflitos, melhoria das condições ambientais de trabalho, melhor utilização das tecnologias em saúde, valorização da participação do trabalhador e de ações/projetos de humanização, além de acompanhamento psicológico ${ }^{(26,40)}$.

Entre exemplos de intervenção protetiva foram relatadas ações de (re)educação alimentar e nutricional aos profissionais sedentários ${ }^{(39)}$, e ainda estratégias voltadas para a biossegurança e a imunização ${ }^{(35,42)}$.

\section{Discussão}

Dentre os estudos que abordaram a relação do adoecimento e do estresse com o ambiente de trabalho, destacaram-se aqueles que trataram da importância do adoecimento psíquico, por vezes relacionado a fatores da organização do trabalho, especialmente de conflitos em relações hierárquicas no ambiente de trabalho. Os problemas psíquicos integram-se à rotina do trabalhador da APS e se alimentam de sentimentos de incapacidade, impotência e falta de recursos. Os resultados dos estudos indicaram índices preocupantes desse tipo de adoecimento e das insuficientes intervenções a curto ou médio prazo. De acordo com a Organização Panamericana da Saúde (OPAS), os transtornos mentais tiveram um aumento de 15\% entre 2005 e 2015, e serão a principal causa de incapacidade laboral nos próximos anos ${ }^{(43-44)}$.

Entende-se que o comprometimento dos trabalhadores com suas funções e objetivos acarretam excessivas preocupações e adoecimento, sem que compensações materiais sejam condizentes com ganhos, remuneração, planos de cargos e carreiras. Estas situações, aliadas a tempos de crise econômica e desemprego, exacerbam a ansiedade e exigem conviver com a exaustão e o esgotamento da saúde mental.
Dentre as condições psíquicas, a síndrome de Burnout tem sido relatada em estudos com diferentes profissionais de saúde. Sua relação com a rotatividade nos serviços enseja reflexão sobre esse contexto, seja por iniciativas pessoais ou pela gestão dos serviços. Na APS brasileira, a rotatividade de profissionais tem sido alta, e as justificativas variam conforme a região, a estruturação da rede de serviços e a modalidade de contratação, dentre outras explicações ${ }^{(45-46)}$.

O maior desafio para o enfrentamento ao sofrimento psíquico e mental recai na detecção precoce da presença de sofrimento (estresse, ansiedade, depressão, estafa, Burnout, dentre outros), antes que consequências duradouras se instalem, inclusive a desmobilização, descrença e resistência à mudança/inovação no processo de trabalho ${ }^{(24)}$.

Fica evidente que o estresse no ambiente de trabalho na APS é um fenômeno complexo, que resulta em desgastes para o trabalhador e incide na perda de qualidade do cuidado e no adoecimento do profissional.

Sob a perspectiva da ética, pesquisas brasileiras relativas ao processo de distresse moral destacam sua multicausalidade, em razão de obstáculos e constrangimentos presentes nos ambientes de trabalho, que impedem o trabalhador de agir conforme seu julgamento moral, alterando sua percepção de si e a própria experiência ético-moral ${ }^{(47-48)}$.

Além do aspecto psíquico, o trabalhador também adoece fisicamente. As doenças crônicas são frequentemente manifestadas por trabalhadores da saúde. Condições crônicas de saúde incidem silenciosamente integradas ao cotidiano do trabalho e manifestam-se num significativo número de trabalhadores que sofre com problemas cardíacos, circulatórios e/ou respiratórios $^{(49)}$. Entende-se que não se trata de fragmentar os adoecimentos psíquico e físico, mas de exprimir um alerta sobre a inter-relação cíclica entre esses dois efeitos dos ambientes não saudáveis sobre a saúde do trabalhador.

Grande parte dos estudos analisados expressa o aumento da escolaridade e a participação esmagadora do gênero feminino no mercado de 
trabalho em saúde. Embora o estresse não se configure como exclusividade de nenhuma profissão, é enfatizado o trabalho da Enfermagem, pelo excesso de atividades ${ }^{(42)}$, ao que se pode adicionar o acúmulo de funções, a dupla jornada para enfermeiras-mulheres-mães. O cenário brasileiro, ao sustentar uma forte cultura patriarcal e machista, penaliza a mulher com sobrecarga de múltiplas tarefas e responsabilidade, o que ainda é reproduzido pelas próprias mulheres, que relutam em abandonar o controle da criação dos filhos, mantendo a desigualdade na divisão de $\operatorname{tarefas}^{(50)}$.

Se, de um lado, as realidades ainda expõem ambientes de trabalho permeados por conflitos que predispõem ao adoecimento e ao estresse, por outro, observam-se estratégias de enfrentamento ou abrandamento dos efeitos negativos. São alternativas coletivas ou individuais de iniciativa do próprio trabalhador, que busca superar as dificuldades e promover o cuidado de si. Considera-se, após a análise dos estudos desta revisão, que as ações ainda são pontuais e limitadas. A abrangência e amplitude das estratégias de enfrentamento devem envolver o coletivo dos trabalhadores da APS e ser continuamente promovidas.

Entende-se que as ações para a promoção de ambientes de trabalho saudáveis não podem ser dissociadas de condições concretas de trabalho e de participação dos trabalhadores, nem considerar apenas demandas por produtividade e alcance de métricas, mas devem incluir o bem-estar, a proteção, a segurança e a promoção da saúde do trabalhador.

No tocante ao controle de agravos relacionados ao trabalho, estudo identifica sérios problemas, incluindo a subnotificação de casos agudos e crônicos, ainda influenciada pelo preconceito, desconhecimento e insegurança quanto aos direitos. A maior evidência, nessa dimensão do cuidado à saúde do trabalhador, é o reconhecimento da importância da informação e do acesso aos recursos e mecanismos para a prevenção de agravos, as formas de trabalhar melhor e manejar relações interpessoais, conflitos e dificuldades do ambiente de trabalho. Fica clara, nas ações de vigilância em saúde do trabalhador, a garantia de participação de representantes dos trabalhadores e assessores técnicos de vigilância nos ambientes de trabalho, além do estabelecimento de estratégias de promoção de saúde, mediante negociação com trabalhadores para participação ${ }^{(51)}$.

Ao falar de medidas protetoras ao trabalhador na APS, cabe considerar a complexidade dos territórios; portanto, é necessária a integração de outros setores, como a segurança pública, a organização dos trabalhadores e a educação, especialmente por abranger questões relacionadas às vulnerabilidades sociais ${ }^{(49-50)}$. Essa integração das instâncias, conforme diretrizes de vigilância em saúde do trabalhador no SUS, compreende o apoio matricial e as ações solidárias e complementares entre regiões, estados e municípios, para reforçar as ações nas redes de atenção, na promoção da saúde e na educação em saúde ${ }^{(51-52)}$.

As limitações deste estudo são decorrentes das estratégias implementadas na busca, conforme critérios de inclusão e exclusão, fato que o circunscreve à amostra de uma realidade dada por parâmetros próprios desta revisão.

\section{Conclusão}

A revisão permitiu concluir-se que os elementos constituintes do ambiente de trabalho saudável na APS, relacionados à saúde dos trabalhadores, são geradores de adoecimento e estresse e estão em constante associação ao contexto desse nível de atenção, à gestão política do processo de trabalho na saúde e às vivências subjetivas no trabalho.

Os achados desta investigação podem contribuir para melhorias na atenção à saúde dos trabalhadores da APS, especialmente no que se refere à gestão de pessoas. Partindo da premissa que, da colaboração entre pesquisa, ensino e gestão, sob embasamentos científicos da área de Enfermagem, ocorre provocação para a transformação a médio e longo prazo desses ambientes de saúde, o resultado também pode repercutir em melhorias da assistência aos usuários.

Diante de situações desafiadoras para a constituição de ambientes de trabalho saudáveis, os trabalhadores vislumbram estratégias 
de enfrentamento dos limites, para a proteção coletiva e individual. O conhecimento como produto de iniciativas de educação parece ser peça-chave para a mobilização transformadora dos ambientes. Entretanto, o processo é complexo, exige diálogo entre todos os atores, intersetorialidade e consolidação de políticas públicas de saúde do trabalhador.

\section{Colaborações:}

1 - concepção, projeto, análise e interpretação dos dados: Lenna Eloisa Madureira Pereira, Flavia Regina Souza Ramos e Paola da Silva Diaz; 2 - redação do artigo e revisão crítica relevante do conteúdo intelectual: Lenna Eloisa Madureira Pereira, Flavia Regina Souza Ramos e Laura Cavalcanti de Farias Brehmer;

3 - aprovação final da versão a ser publicada: Lenna Eloisa Madureira Pereira, Flavia Regina Souza Ramos, Laura Cavalcanti de Farias Brehmer e Paola da Silva Diaz.

\section{Referências}

1. Organização Mundial da Saúde. Ambientes de trabalho saudáveis: um modelo para ação. Para empregadores, trabalhadores, formuladores de política e profissionais. Tradução do Serviço Social da Indústria. Brasília (DF): SESI/DN; 2010 [cited 2020 Jan 10]. Available from: https://www.who.int/ occupational_health/ambientes_de_trabalho.pdf

2. Alves DFS, Guirardello EB. Ambiente de trabalho da enfermagem, segurança do paciente e qualidade do cuidado em hospital pediátrico. Rev Gaúcha Enferm (Online). 2016;37(2):e58817. DOI: http://doi.org/10.1590/1983-1447.2016.02. 58817

3. Post Sennehed C, Gard G, Holmberg S, Stigmar K, Forsbrand M, Grahn B. "Blue flags", development of a short clinical questionnaire on work-related psychosocial risk factors - a validation study in primary care. BMC Musculoskelet Disord. 2017;18(318):1-11. DOI: 10.1186/s12891-017-1677-z

4. Scherer MDA, Oliveira NA, Pires DEP, Trindade LL, Gonçalves ASR, Vieira M. Aumento das cargas de trabalho em técnicos de enfermagem na atenção primária à saúde no Brasil. Trab educ saúde (Online). 2016;14(Suppl 1):
89-104. DOI: https://doi.org/10.1590/1981-7746sol00030

5. Trifunovic N, Jatic Z, Kulenovic AD. Identification of Causes of the Occupational Stress for Health Providers at Different Levels of Health Care. Med Arch. 2017;71(3):169-72. DOI: 10.5455/ medarh.2017.71.169-172

6. Brasil. Ministério da Saúde. Portaria nº 2.436, de 21 de setembro de 2017. Aprova a Política Nacional de Atenção Básica, estabelecendo a revisão de diretrizes para a organização da Atenção Básica, no âmbito do SUS. Brasília (DF): 2017 [cited 2020 Jan 10]. Available from: https:// bvsms.saude.gov.br/bvs/saudelegis/gm/2017/ prt2436_22_09_2017.html

7. Mendes KDS, Silveira RCCP, Galvão CM. Revisão integrativa: método de pesquisa para incorporação de evidências na saúde e na enfermagem. Texto contexto - enferm. 2008;17(4):758-64. DOI: https://doi.org/10.1590/ S0104-07072008000400018

8. Peralta N, Godoi Vasconcelos AG, Griep RH, Miller L. Validez y confiabilidad del Índice de Capacidad para el Trabajo en trabajadores del primer nivel de atención de salud en Argentina. Salud Colectiva. 2012 [cited 2019 Aug 14];8(21): 161-73. Available from: https://www.arca.fiocruz. br/handle/icict/16310

9. Halcomb E, Ashley C. Australian primary health care nurses most and least satisfying aspects of work. J Clin Nurs. 2017 Feb;26(3-4):535-45. DOI: 10.1111/jocn.13479

10. Abbas MAF, Fiala LA, Rahman AGA, Fahim AE. Epidemiology of workplace violence against nursing staff in ismailia governorate, Egypt. J Egypt Public Health Assoc. 2010 [cited 2019 Aug 14];85(1-2):29-43. Available from: https:// pubmed.ncbi.nlm.nih.gov/21073846/

11. Pinto ACS, Almeida MI, Pinheiro PNC. Análise da susceptibilidade às doenças imunopreveníveis em profissionais de saúde a partir do status vacinal. Rev Rene. 2011 jan/mar [cited 2019 Aug 14];12(1):104-10. Available from: http:// www.periodicos.ufc.br/rene/article/view/4162/ 3230

12. Dias MDA. Compreender o trabalho na Atenção Primária à Saúde para desenvolver ações em Saúde do Trabalhador: o caso de um município de médio porte. Rev bras saúde ocup. 2013 Jun;38(127):69-80. DOI: 10.1590/ S0303-76572013000100010 
13. Bertussi VC, Junqueira MABB, Giuliani CD, Calçado RM, Miranda FJS, Santos MA, et al. Substâncias psicoativas e saúde mental em profissionais de enfermagem da Estratégia Saúde da Família. Rev Eletr Enferm. 2018;20(2021):1-11. DOI: $10.5216 /$ ree.v20.47820

14. González Jara MA, Mora Hidalgo A, Avalos Gulin JC, Albiach ML, Ortiz LM, Monserrat PT, et al. Exposure of health workers in primary health care to glutaraldehyde. J Occup Med Toxicol. 2013;8(31):1-6. DOI: 10. 1186/1745-6673-8-31

15. Siegrist J, Shackelton R, Link C, Marceau L, Knesebeck O, McKinlay J. Work stress of primary care physicians in the US, UK and German health care systems. Soc Sci Med. 2010;71(2):298-304. DOI: 10.1016/j.socscimed.2010. 03.043

16. Oliveira LP, Camargo FC, Iwamoto HH. Violência relacionada ao trabalho das equipes de saúde da família. Rev enferm atenção saúde. 2013 [cited 2019 Aug 14];2(2 NEsp):46-56. Available from: http://seer.uftm.edu.br/revistaeletronica/index. php/enfer/article/view/388

17. Gómez-Gascón T, Martín-Fernández J, GálvezHerrer M, Tapias-Merino E, Beamud-Lagos M, Mingote-Adán JC, et al. Effectiveness of an intervention for prevention and treatment of burnout in primary health care professionals. BMC Fam Pract. 2013 Nov 17;14:173. DOI: 10.1186/1471-2296-14-173

18. Costa PCP, Garcia APRF, Toledo VP. Expectativa de enfermeiros brasileiros acerca do acolhimento realizado na atenção primária em saúde. Rev salud publica. 2017 Feb;18(5):746-55. DOI: 10. 15446/rsap.v18n5.45304

19. Hongxia Guo RN, Chunping Ni RN, Changqing Liu RN, Jiping Li BN, Suzhen Liu MSN. Perceived job stress among community nurses: A multicenter cross-sectional study. Int J Nurs Pract. 2019 Feb;25(1):e12703. DOI: 10.1111/ijn. 12703

20. Silva MO, Peixoto DA, Nóbrega TBT. Grupos de encontro na estratégia saúde da família: uma atenção ao trabalhador de saúde. Rev APS. $2013 \mathrm{abr} /$ jun [cited 2019 Aug 14];16(2):197-201. Available from: https://periodicos.ufff.br/index. php/aps/article/view/15034/7961

21. Cipriano FG, Ferreira LP, Servilha EAM, Marsiglia RMG. Relação entre distúrbio de voz e trabalho em um grupo de Agentes Comunitários de Saúde. CoDAS. 2013 [cited 2019 Aug 14];25(6):548-56. Available from: https://www. sanarmed.com/artigos-cientificos/relacao-entredisturbio-de-voz-e-trabalho-em-um-grupo-deagentes-comunitarios-de-saude

22. Silveira SLM, Câmara SG, Amazarray MR. Preditores da Síndrome de Burnout em profissionais da saúde na atenção básica de Porto Alegre/RS. Cad saúde colet. 2014;22(4): 386-92. DOI: https://doi.org/10.1590/1414-462X 201400040012

23. Scherer MDA, Oliveira CI, Carvalho WMES, Costa MP. Specialization training courses on Family Health: what can training change in the work? Interface (Botucatu, Online). 2016;20(58):691-702. DOI: https://doi.org/10.1590/ $1807-57622015.0020$

24. Silva SCPS, Nunes MAP, Santana VR, Reis FP, Machado Neto J, Lima SO. A síndrome de burnout em profissionais da rede de atenção primária à saúde de Aracaju, Brasil. Ciênc Saúde Colet. 2015;20(10):3011-20. DOI: https://doi.org/ 10.1590/1413-812320152010.19912014

25. Speroni KS, Fruet IMA, Dalmolin GL, Lima SBS. Percepções dos agentes comunitários de saúde: contribuições para a gestão em saúde. Rev Cuid (Bucaramanga. 2010). 2016;7(2):1325-37. DOI: https://doi.org/10.15649/cuidarte.v7i2.338

26. Pegoraro PBB, Schaefer R, Zoboli ELCP. Psychic and moral exhaustion in primary care workers. Rev Esc Enferm USP. 2017;51:e03257. DOI: https://doi.org/10.1590/S1980-220X2016035203257

27. Costa FM, Martins AMEBL, Lima CA, Rodrigues QF, Santos KKF, Ferreira RC. Fatores associados à verificação da imunização pós-vacinação contra hepatite $\mathrm{B}$ entre trabalhadores da Atenção Primária. Cad saúde colet. 2017;25(2):192-200. DOI: https://doi. org/10.1590/1414-462X201700020136

28. Zhang M, Yang R, Wang W, Gillespie J, Clarke S, Yan F. Job satisfaction of urban community health workers after the 2009 healthcare reform in China: a systematic review. Int J Qual Health Care. 2016;28(1):14-21. DOI: 10.1093/intqhe/mzv111

29. Nehmy RMQ, Dias EC. Os caminhos da Saúde do Trabalhador: para onde apontam os sinais? Rev Méd Minas Gerais. 2010 [cited 2019 Aug 14];20(2 Suppl 2):S13-S23. Available from: http:// rmmg.org/artigo/detalhes/1030 
30. Maun A, Nilsson K, Furaker C, Thorn J. Primary healthcare in transition - a qualitative study of how managers perceived a system change. BMC Health Serv Res. 2013;13:382. DOI: 10.1186/1472-6963-13-382

31. Bhatnagar A, Gupta S, Alonge O, George AS. Primary health care workers' views of motivating factors at individual, community and organizational levels: a qualitative study from Nasarawa and Ondo states, Nigeria. Int J Health Plann Manage. 2016;32(2):217-33. DOI: 10.1002/ hpm.2342

32. Fisekovic Kremic MB, Terzic-Supic ZJ, Santric-Milicevic MM, Trajkovic GZ. Encouraging employees to report verbal violence in primary health care in Serbia: a cross-sectional study. Zdr Varst. 2017;56(1):11-7. DOI: 10.1515/ sjph-2017-0002

33. Rule J, Dunston R, Solomon N. Learning and change in the redesign of a primary health care initiative. J Workplace Learn. 2016;28(7):451-67. DOI: 10.1108/JWL-09-2015-0069

34. Silva CCS, Rodrigues LMC, Silva VKBA, Silva ACO, Silva VLA, Martins MO. Percepção da enfermagem sobre condições de trabalho em unidades de saúde da família na Paraíba - Brasil. Rev Eletr Enf. 2013;15(1):205-14. DOI: https://doi. org/10.5216/ree.v15i1.15074

35. Pessoa VM, Rigotto RM, Arruda CAM, Machado MFAS, Machado MMT, Bezerra MGV. Action research: methodological proposal for action planning in primary care services in the context of environmental health and occupational health. Interface (Botucatu). 2013;17(45):301-14. DOI: https://doi.org/10.1590/ S1414-32832013005000004

36. Costa JO, Sousa MNA, Feitosa ANA, Feitosa MO, Assis EV, Custódio PP. Gestão de conflitos: estratégias adotadas em unidade básica de saúde. Rev adm Saúde. 2013 [cited 2019 Aug 14];15(61):134140. Available from: https://pesquisa. bvsalud.org/portal/resource/pt/lil-745015

37. Carreiro GSP, Ferreira Filha MO, Lazarte R, Silva AO, Dias MD. O processo de adoecimento mental do trabalhador da Estratégia Saúde da Família. Rev Eletr Enferm. 2013;15(1):146-55. DOI: https://doi.org/10.5216/ree.v15i1.14084

38. Duffrin C, Diaz S, Cashion M, Watson R, Cummings D, Jackson N. Factors associated with placement of rural primary care physicians in
North Carolina. South Med J. 2014;107(11):728-33. DOI: 10.14423/SMJ.0000000000000196

39. Ojakaa D, Olango S, Jarvis J. Factors affecting motivation and retention of primary health care workers in three disparate regions in Kenya. Hum Resour Health. 2014;12:33. DOI: 10.1186/1478-4491-12-33

40. Rao KD, Ryan M, Shroff Z, Vujicic M, Ramani S, Berman P. Rural clinician scarcity and job preferences of doctors and nurses in India: a discrete choice experiment. PLoS One. 2013;8(12):e82984. DOI:10.1371/journal. pone.0082984

41. Cahú GRP, Costa SFG, Costa ICP, Batista PSS, Batista JBV. Situações de assédio moral vivenciadas por enfermeiros no ambiente de trabalho. Acta paul enferm. 2014;27(2):151-6. DOI: https://doi. org/10.1590/1982-0194201400027

42. Holmes ES, Santos SR, Farias JA, Costa MBS. Síndrome de burnout em enfermeiros na atenção básica: repercussãona qualidade devida. Rev Pesqui (Univ Fed Estado Rio J., Online). 2014;6(4):138495. DOI: http://doi.org/10.9789/2175-5361

43. Organização Pan-Americana da Saúde, Organização Mundial da Saúde - Américas. Com depressão no topo da lista de causas de problemas de saúde, OMS lança a campanha "Vamos conversar" [Internet]. Brasília (DF); 2017 [cited 2020 Jan 10]. Available from: https:// www paho.org/bra/index.php?option $=\mathrm{com}_{-}$ content\&view $=$ article $\&$ id $=5385$ : com-depressao - notopo-da-lista-de-causas-de-problemas-de-saudeoms-lanca-a-campanha-vamos-conversar \&Itemid=839

44. Associação Nacional de Medicina do Trabalho. OMS: empresas devem promover saúde mental de funcionários [Internet]. Rio de Janeiro (RJ); 2017 [cited 2020 Jan 10]. Available from: https://www.anamt.org.br/portal/2017/10/11/ oms-empresas-devem-promover-saude-mentalde-funcionarios/

45. Willard-Grace R, Knox M, Huang B, Hammer H, Kivlahan C, Grumbach K. Burnout and Health Care Workforce Turnover. Ann Fam Med. 2019;17(1):3641. DOI: $10.1370 / \mathrm{afm} .2338$

46. Pierantoni CR, Vianna CMM, França T, Magnago C, Rodrigues MPS. Turnover of the medical workforce in Brazil. Saúde debate. 2015; 39(106):637-47. DOI: http://doi.org/10.1590/0103110420151060003006 
47. Ramos FRS, Barlem ELD, Brito MJM, Vargas MA, Schneider DG, Brehmer LCF. Marco conceitual para o estudo do distresse moral em enfermeiros. Texto contexto-enferm. 2016;25(2):e4460015. DOI: https://doi.org/10.1590/ 0104-07072016004460015

48. Barth PO, Ramos FRS, Barlem ELD, Dalmolin GL, Schneider DG. Validation of a moral distress instrument in nurses of primary health care. Rev Latino-Am Enfermagem. 2018;26:e3010. DOI: $10.1590 / 1518-8345.2227 .3010$

49. Santos AC, Hoppe AS, Krug SBF. Agente Comunitário de Saúde: implicações dos custos humanos laborais na saúde do trabalhador. Physis. 2018;28(4):e280403. DOI: https://doi. org/10.1590/S0103-73312018280403

50. Costa FA. Mulher, trabalho e família: os impactos do trabalho na subjetividade da mulher e em suas relações familiares. Pretextos - Rev Grad Psicol PUC Minas. 2018 [cited 2020 Jan 10];
3(6):434-52. Available from: http://periodicos. pucminas.br/index.php/pretextos/article/view/ 15986

51. Brasil. Ministério da Saúde. Secretaria de Vigilância em Saúde. Diretrizes de implantação da Vigilância em Saúde do Trabalhador no SUS. Brasília (DF); 2014 [cited 2020 Jan 10]. Available from: https://renastonline.ensp.fiocruz.br/sites/ default/files/arquivos/recursos/Diretrizes-deimplantacao-da-Vigilancia-em-Saude-doTrabalhador-no-SUS.pdf

52. Fernandes DM, Marcolan JF. Trabalho e sintomatologia depressiva em enfermeiros da Estratégia de Saúde da Família. SMAD, Rev Eletrônica Saúde Mental Alcool Drog. 2017;13(1):37-44. DOI: https://doi.org/10.11606/ issn.1806-6976.v13i1p37-44

Recebido: 27 de julho de 2020

Aprovado: 20 de junho de 2021

Publicado: 26 de novembro de 2021

A Revista Baiana de Enfermagem utiliza a Licença Creative Commons - Atribuição-NãoComercial 4.0 Internacional.

https://creativecommons.org/licenses/by-nc/4.0/

Este artigo é de acesso aberto distribuído sob os termos da Licença Creative Commons (CC BY-NC).

Esta licença permite que outros remixem, adaptem e criem a partir do seu trabalho para fins não comerciais. Embora os novos trabalhos tenham de lhe atribuir o devido crédito e não possam ser usados para fins comerciais, os usuários não têm de licenciar esses trabalhos derivados sob os mesmos termos. 